\title{
Atemnot und COVID-19: Ein Aufruf zu mehr Forschung
}

\author{
Lisa Hentsch $^{a}$ Sara Cocetta ${ }^{b}$ Gilles Allalic, d Isabelle Santana ${ }^{e}$ Rowena Eason ${ }^{f}$ \\ Emily Adam ${ }^{g}$ Jean-Paul Janssens ${ }^{\text {h }}$
}

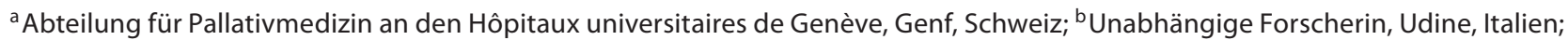
'Abteilung für Neurologie, Hôpitaux universitaires de Genève und Medizinische Fakultät der Universität Genf, Genf, Schweiz; ${ }^{d}$ Division of Cognitive and Motor Aging, Department of Neurology, Albert Einstein College of Medicine, Yeshiva University, Bronx, NY, USA; ${ }^{e}$ Federal University of Bahia, Salvador, Brasilien; ${ }^{f}$ Phyllis Tuckwell Hospice Care, Surrey, Vereinigtes Königreich; gUnabhängige Forscherin, London, Vereinigtes Königreich; ${ }^{h}$ Abteilung für Lungenkrankheiten, Hôpitaux universitaires de Genève, Genf, Schweiz
\end{abstract}

\section{Schlüsselwörter}

Coronavirus-Krankheit 2019 · Schweres akutes Atemwegssyndrom .

Coronavirus Typ 2 · Atemnot · Dyspnoe · Neuroinvasion

\begin{abstract}
Zusammenfassung
Atemnot, auch als Dyspnoe bezeichnet, ist ein häufiges und lähmendes Symptom. In mehreren Berichten wurde die Abwesenheit von Atemnot bei einer Untergruppe von Patienten mit COVID-19 hervorgehoben, die manchmal als «stille» oder «glückliche Hypoxie» bezeichnet wird. Ebenfalls wurde in Berichten erwähnt, dass es an einem klaren Zusammenhang zwischen dem klinischen Schweregrad der Erkrankung und der von den Patienten berichteten Schwere der Atemnot fehlt. Die zerebralen Komplikationen von COVID-19 sind weitgehend nachgewiesen, mit einer hohen Prävalenz akuter Enzephalopathien, die möglicherweise die Verarbei-
\end{abstract}

tung afferenter Signale oder die absteigende Modulation von Atemnotsignalen beeinträchtigen könnte. In dieser Übersichtsarbeit möchten wir die an der Atemnot beteiligten Mechanismen hervorheben und die Pathophysiologie von COVID-19 und den bekannten Auswirkungen der Erkrankung auf die Interaktion von Gehirn und Lunge zusammenfassen. Anschließend stellen wir Hypothesen für die Veränderung der Wahrnehmung von Atemnot bei COVID-19-Patienten auf und schlagen Möglichkeiten vor, mit denen dieses Phänomen weiter erforscht werden könnte.

(c) 2021 S. Karger AG, Basel

\section{Einleitung}

Atemnot, auch als Dyspnoe bezeichnet, ist ein häufiges und belastendes Symptom. Sie wurde als das subjektive Erleben von Atembeschwerden definiert, die aus qualitativ verschiedenen Empfindungen von unterschiedlicher Intensität besteht [1]. Viele verschiedene Erkrankungen, beispielsweise Lungenerkrankungen, chronische Herzinsuffizienz und neurodegenerative Erkrankungen, verursachen ähnliche subjektive Empfindungen. Daher wurde bereits die Hypothese aufgestellt, dass unabhängig von der zugrunde liegenden Ursache eine gemeinsame Leitungs- bahn des zentralen Nervensystems an der Wahrnehmung von Dyspnoe beteiligt sein könnte [1].

Ende 2019 wurde erstmals in Wuhan (China) über eine neue Atemwegsinfektion, die Coronavirus-Krankheit 2019 (coronavirus disease 2019, COVID-19), auch: schweres akutes Atemwegssyndrom-Coronavirus Typ 2 (severe acute respiratory syndrome coronavirus 2, SARS-CoV-2), berichtet, die von einem neuen Coronavirus ausgelöst wird. Obwohl die Prävalenz von schweren Erkrankungsverläufen hoch war, war die Prävalenz von Dyspnoe bei COVID-19-Patienten im Vergleich zu anderen Coronaviren wie dem schweren akuten Atemwegssyndrom (SARS)- 
CoV (SARS-CoV) oder dem Middle East Respiratory Syndrome (MERS)-CoV (MERS-CoV) relativ gering [2]. Das Ausmaß der Dyspnoe scheint bei COVID-19-Patienten erheblich zu variieren und reicht von 18,6 bis 59\% [3, 4]. In einer systematischen Übersichtsarbeit und Metaanalyse stellten Rodriguez-Morales et al. [5] eine Dyspnoe-Prävalenz von insgesamt 45,6\% (95\%-Konfidenzintervall (KI): 10,9-80,4\%) fest. In jüngerer Zeit führten Li et al. [6] eine systematische Übersichtsarbeit durch, in die Daten von 1994 Patienten einflossen.

Der Gesamtprozentsatz der Patienten mit Dyspnoe betrug dort 21,9\%. Ein Teil der Unterschiede in der Dyspnoe-Prävalenz innerhalb und zwischen den Studien kann durch Unterschiede bei der Untersuchung und Dokumentation erklärt werden. Möglicherweise hängen sie auch mit der jeweiligen Klinikumgebung zusammen. In einer prospektiven Studie zum klinischen Verlauf von hospitalisierten COVID-19-Patienten stellten Huang et al. [7] eine Atemnotprävalenz von bis zu $92 \%$ bei auf Intensivstationen behandelten Patienten gegenüber 37\% bei Patienten fest, die nicht auf der Intensivstation behandelt wurden. Viele Studien, die in systematische Übersichtsartikel eingeschlossen wurden, sind retrospektiv; in ihnen wurde über Symptome bei der Aufnahme berichtet, die retrospektiv aus den Krankenakten der Patienten ausgelesen wurden $[8,9]$. Wie, wann und von wem die Dyspnoe beurteilt wurde, ist unklar und wird weiter diskutiert.

Obwohl Dyspnoe bei Erwachsenen mit COVID-19 signifikant mit einer erhöhten Sterblichkeit assoziiert ist, (Odds Ratio (OR) $=4,34,95 \%$-KI: [2, 68-7, 05], $p<0,001, I^{2}=69,2 \%, p<0,001$, Random-Effects-Modell) [10], korreliert sie nicht immer mit der Krankheitsschwere. Guan et al. [3] haben in einer retrospektiven Datenanalyse von 1099 Patienten tatsächlich nur bei 18,6\% eine Dyspnoe festgestellt, obwohl 86\% abnorme CT-Aufnahmen und einen niedrigen $\mathrm{PaO}_{2} / \mathrm{FiO}_{2}$-Quotienten aufwiesen. Andere Fallstudien haben ergeben, dass auch bei Patienten mit erhöhtem $\mathrm{PaO}_{2}$ eine sogenannte «stille Hypoxie» beobachtet wurde, die in Kombination mit einem niedrigen $\mathrm{PaO}_{2}$-Wert eigentlich eine Atemnot hervorrufen sollte. Tatsächlich reagiert das Atemzentrum, wie weiter unten erörtert wird, äußerst empfindlich auf einen $\mathrm{CO}_{2}$-Anstieg, was das Atemzeitvolumen rasch erhöht und allgemeine Atembeschwerden verursacht [11]. Dies wurde bei Patienten mit und ohne Komorbiditäten beobachtet [11].

Warum bestimmte Patienten eine «stille» oder «glückliche Hypoxie» zeigen, ist zu diesem Zeitpunkt noch unklar. Bestimmte Patientengruppen, insbesondere ältere Menschen und Diabetespatienten, sind aus Gründen, die im weiteren Verlauf erörtert werden, möglicherweise einem höheren Risiko ausgesetzt. Bis heute wurden keine Patientenmerkmale oder Komorbiditäten eindeutig mit einer veränderten Wahrnehmung von Atemnot bei COVID-19 in Verbindung gebracht. Liet al. [12] haben darauf hingewiesen, dass das neuroinvasive Potenzial von SARS-CoV-2 durch seine Wirkung auf den Hirnstamm und das medulläre kardiorespiratorische Zentrum bei infizierten Patienten für die veränderte Wahrnehmung von Atemnot verantwortlich sein könnte. Andere haben hingegen nahegelegt, dass die Abwesenheit von Atemnot eher auf die kortikalen anstatt auf die subkortikalen
Strukturen zurückzuführen sei [13]. Eine verminderte Wahrnehmung von Atemnot könnte auch an der Veränderung der peripheren Strukturen wie der veränderten Wahrnehmung der Muskelanstrengung, einer verminderten thorakalen Compliance oder einem veränderten Input von den Mechanorezeptoren aus dem Atmungstrakt und der Brustwand [14] liegen. Ebenfalls wurde die Hypothese aufgestellt, dass SARS-CoV-2 durch seine direkte Wirkung auf den Vagusnerv mechanische und chemische Rezeptoren an diesem Nerv hemmt, die im Allgemeinen für eine Exazerbation der Dyspnoe verantwortlich sind $[15,16]$.

In dieser Übersichtsarbeit fassen wir die aktuelle Evidenz zu den Mechanismen zusammen, die an der Modulation der Atemnot von COVID-19-Patienten beteiligt sind. Anschließend stellen wir Hypothesen zu den möglichen Ursachen einer verminderten Wahrnehmung von Atemnot bei diesen Patienten auf und weisen auf weitere Forschungsgebiete hin, um nicht nur das Wissen über COVID-19, sondern auch unsere Kenntnisse der Mechanismen zu verbessern, die der Atemnot im Allgemeinen zugrunde liegen.

\section{Mechanismen bei der Wahrnehmung von Atemnot}

Verglichen mit Symptomen wie Schmerz sind die pathophysiologischen Mechanismen der Atemnot weniger umfassend bekannt [17]. Sie beinhalten die Aktivierung von Rezeptoren in der Brustwand, im Lungenparenchym und in den Atemwegen sowie die Stimulation des Atemzentrums im Hirnstamm durch periphere und zentrale Chemorezeptoren [1].

Dyspnoe ist wie Schmerz ein subjektiv empfundenes Symptom, was die Messung seines Schweregrades schwierig gestaltet. Schmerz und Atemnot werden beide in Teilen vom limbischen System verarbeitet [18]. Diese Symptome machen den Körper auf bedrohliche Zustände und den möglichen Verlust der Homöostase aufmerksam, wodurch sie die Person dazu motivieren, Hilfe zu suchen und adaptive Verhaltensweisen umzusetzen [19]. Allerdings empfindet der Mensch im frühen Lebensalter verhältnismäßig häufig Schmerzen und ist dadurch in der Lage, diese zu erkennen und zu beschreiben. Atemnot ist hingegen ein seltener auftretendes Symptom, und der Umstand, dass dieses zuvor nicht erlebt wurde, könnte seine Erkennung schwieriger gestalten [19]. Das Erleben von Dyspnoe beinhaltet sensorische und affektive Dimensionen [17]. Die Schwere (sensorische Intensität) und das unangenehme Empfindungsgefühl (affektive Intensität) der Dyspnoe werden in verschiedenen Hirnarealen verarbeitet [17]. An der Schwere der Dyspnoe sind das laterale Thalamussystem und der primäre sensorische Kortex beteiligt [20]. Am unangenehmen Empfindungsgefühl der Dyspnoe sind eine Aktivierung des limbischen Systems, die rechte vordere Inselrinde und die Amygdala beteiligt [20].

In der Lunge und in den unteren Atemwegen übertragen unmyelinisierte C-Fasern und myelinisierte $\mathrm{A} \delta$-Fasern mit kleinem Durchmesser über die afferenten Vagusnerven mechanische und chemische Signale an den Nucleus des Tractus solitarius im Hirnstamm. Diese Fasern werden nur aktiviert, wenn schädliche Reize dies auslösen; daher gelten sie als nozizeptiv [21]. Nachdem 
diese Fasern im Nucelus des Tractus solitarius zusammengelaufen sind, werden sie an das Gehirn weitergeleitet, genauer gesagt an den somatosensorischen Kortex und in andere Regionen des Gehirns, die an der Interpretation dieser Signale beteiligt sind, wodurch die Wahrnehmung von Atemnot erzeugt wird [21].

Der Kohlendioxidspiegel wird im Wesentlichen durch medulläre Chemorezeptoren zentral überwacht, jedoch sind auch periphere Chemorezeptoren an diesem Prozess beteiligt. Der Sauerstoffspiegel im Blut wird jedoch peripher durch Chemorezeptoren überwacht, die sich in den Glomera aortica und im Glomus caroticum befinden.

Diese peripheren Signale werden vom Nervus glossopharyngeus und vom Vagusnerv an den Nucleus des Tractus solitarius übertragen, wo sie wiederum an höhere Hirnstrukturen weitergeleitet werden [21].

Diese Reize scheinen unabhängig vom objektiven Atmungsstatus, jedoch in Abhängigkeit von den vergangenen Erlebnissen, Emotionen und Überzeugungen der jeweiligen Person unterschiedlich wahrgenommen und interpretiert zu werden [22-24]. Tatsächlich kann sich die Wahrnehmung von Dyspnoe von Person zu Person erheblich unterscheiden $[25,26]$.

Das individuelle Verhalten einer Person wird von ihrer affektiven Reaktion auf das Symptom bestimmt, was in einigen Fällen zu einer nicht ausreichenden Wahrnehmung der Dyspnoe mit folglicher Nichterkennung der Schwere der Erkrankung führen kann [27]. Eine häufigere Angabe von Dyspnoe korrespondiert häufig mit stärkerer Angst und führt zudem zu häufigeren Krankenhauseinweisungen $[25,26]$.

Die sensorischen und affektiven Dimensionen der Atemnot erstrecken sich auf unterschiedliche Nervenbahnen. Informationen zu affektiven Aspekten von Atemnot werden im limbischen System übermittelt, wandern von den vagalen Afferenzen in der Lunge und in den Atemwegen zum Hirnstamm und zur Medulla, werden anschließend zur Amygdala und in den medialen dorsalen Bereich des Thalamus weitergeleitet und kommen schließlich in der Inselrinde und im zingulären Kortex an. Hyperkapnie ist ein starker Reiz zur Aktivierung des limbischen Systems, wodurch die affektive Dimension der Dyspnoe erhöht wird [28].

Die affektiven Dimensionen von Atemnot könnten einen starken Einfluss auf die subjektive Bewertung des Atemnotgefühls haben und zu einer Überwahrnehmung des Symptoms führen [29]. Negative Emotionen haben einen großen Einfluss auf die Wahrnehmung von Atemnot, während positive Emotionen die Intensität der Wahrnehmung von Atemnot verringern [30].

Die verhaltensbezogenen Auswirkungen von Atemnot haben Konsequenzen für die täglichen Aktivitäten einer Person und beeinträchtigen das psychische Wohlbefinden sowie das soziale Leben [31]. Mit der Vermeidung von körperlicher Aktivität, um die Dyspnoe zu verringern, beginnt ein Teufelskreis, der möglicherweise zu einer Verschlechterung des allgemeinen Leistungsstatus und zur Pflegebedürftigkeit sowie zu sozialer Isolation, vermindertem Selbstwertgefühl und Angst führt. Episoden plötzlicher Atemnot können als Nahtoderfahrung wahrgenommen werden und hinterlassen bei Patienten Symptome einer posttraumatischen Belas- tungsstörung [31]. Soziale Einschränkungen, wie eine Einschränkung von Aktivitäten, die Angst vor einer Ansteckung, der Verlust des Arbeitsplatzes, finanzielle Probleme und die Belastung von Pflegepersonen könnten im Hinblick auf Patienten, die infolge von COVID-19 an Atemnot leiden, zu bedeutsamen Problemen werden.

\section{Mitursachen der Wahrnehmung von Atemnot}

Es gibt Hinweise darauf, dass ältere Menschen tendenziell weniger von Dyspnoe berichten [27]. Der Alterungsprozess führt nicht nur zu physiologischen Veränderungen bei den Sinnesorganen, etwa bei denjenigen, die am Hören und Riechen beteiligt sind, sondern wirkt sich auch auf die peripheren Strukturen aus. Beispielsweise werden Veränderungen an den peripheren Rezeptoren im Glomus caroticum mit einer physiologischen Denervierung in Verbindung gebracht, die mit dem Alter auftritt [32]. Es wurde gezeigt, dass die periphere Wahrnehmung von Hyperkapnie, die Reaktion auf Hypoxie sowie die Wahrnehmung zusätzlicher restriktiver oder elastischer Belastungen bei gesunden älteren Personen herabgesetzt sind [27, 33, 34].

Auch psychologische Faktoren spielen bei der Wahrnehmung und Angabe von Atemnot bei älteren Menschen eine Rolle. Unter der Bezeichnung «Positiv-Effekt» wurde beispielsweise über die Tendenz von älteren Menschen berichtet, ihre Aufmerksamkeit von einer negativen Situation abzuziehen und sich stattdessen auf positive Informationen zu konzentrieren, um ihr Wohlbefinden zu erhalten [27]. Die Angst vor Einsamkeit und vor einem Krankenhausaufenthalt kann ebenfalls dazu beitragen; genauso wie eine geringe soziale Unterstützung [27].

Diabetes ist eine häufige Komorbidität bei COVID-19-Patienten [35-37] und steht zudem mit einem erhöhten Risiko für schwere Krankheitsverläufe und eine Aufnahme auf der Intensivstation sowie mit einer erhöhten Sterblichkeit in Verbindung [38]. Die Gründe für die erhöhte Schwere des Krankheitsverlaufs bei Diabetikern hängen mit mehreren Faktoren zusammen, unter anderem mit einer beeinträchtigten Immunantwort, einer verringerten Virenabwehr, einer Prädisposition für Hyperinflammation, einer erhöhten Zytokinausschüttung und einer kardiovaskulären Begleiterkrankung [39, 40].

Diabetes beeinträchtigt nicht nur das Herz-Kreislauf-System und die Nierenfunktion, sondern führt auch zu Schäden am Lungengewebe, auch wenn dieser Zusammenhang noch nicht vollständig verstanden wird [41]. Zu den Mechanismen, die an der Lungenfunktionsstörung beteiligt sind, gehören die Glykosylierung von Lungengewebeproteinen als Folge einer Hyperglykämie, oxidativer Stress, Veränderungen des Bindegewebes, die Beeinträchtigung von Surfactant sowie Schäden an den alveolären Kapillaren und anderen Lungengefäßen [41]. Diese Mechanismen beeinträchtigen die Lungenfunktion und die pulmonale Diffusionskapazität. Eine Verdickung der Basallamina der Lunge, die bei Autopsien von Diabetikern festgestellt wurde, trägt wahrscheinlich zu einer herabgesetzten Lungendiffusion bei, während Gefäßschäden zu einer endothelialen Dysfunktion führen [42]. Eine Verringerung der mukoziliären Clearance erhöht auch die Infek-
Kompass Pneumol 2022;10:6-14 DOI: $10.1159 / 000521460$ 
tionsanfälligkeit [41]. Die Lungenfunktion ist bei Diabetikern auch wegen einer autonomen Neuropathie in Mitleidenschaft gezogen, die zu einer beeinträchtigten Reaktion auf eine Hypoxämie führen kann [41]. Dies kann dazu führen, dass erst mit einer zeitlichen Verzögerung von einer Dyspnoe berichtet wird. Es ist wahrscheinlich, dass eine SARS-CoV-2-Infektion bereits vorhandene diabetische Entzündungsmechanismen und Gefäßschäden verschlimmert, was zu den häufigeren ungünstigen Verläufen bei Diabetikern beiträgt.

\section{Pathophysiologie von COVID-19}

$\mathrm{Zu}$ den radiologischen Befunden bei CT-Aufnahmen von COVID-19-Patienten gehören Lungenentzündungen und Milchglastrübungen als Folge einer systemischen und lokalisierten Entzündung [43]. Bei älteren Patienten zeigt sich unter anderem aufgrund einer erhöhten Prävalenz von Komorbiditäten wie Bluthochdruck, Diabetes und zerebrovaskulären sowie HerzKreislauf-Erkrankungen ein schlechteres klinisches Bild [5]. Die Sterblichkeitsrate wird bei den über 50 -Jährigen mit 8,3\% und bei den über 80 -Jährigen mit 18,8\% angegeben [44]. Insbesondere zerebrovaskuläre sowie Herz-Kreislauf-Erkrankungen und Diabetes sind wichtige Prädiktoren für ungünstige Verläufe [45]. Es gibt drei mögliche Hauptmechanismen, durch die SARSCoV-2 die Atemnot modulieren könnte: Entzündungen in den Alveolen und im Lungengewebe, Thrombosen und damit in Zusammenhang stehende Mikrogerinnsel sowie eine Neuroinvasion.

\section{Entzündung in den Alveolen und im Lungengewebe}

Entzündungen in den Alveolen und im Lungengewebe sind für eine Beeinträchtigung der Gasdiffusionskapazität und einen intrapulmonalen Shunt verantwortlich, was zu einer Hypoxämie mit Normo- oder Hypokapnie führt [46]. Bei Autopsiebefunden von Patienten mit bestätigter COVID-19-Erkrankung wurden diffuse Schäden an den Alveolen mit schwerer Kapillarstauung, Ödemen und alveolärer Hämorrhagie identifiziert [47]. Weitere Befunde sind Pneumozytennekrosen, eine Bildung hyaliner Membranen und Thromben aus plättchenreichem Fibrin [48] Durch von SARS-CoV-2 verursachte Entzündungen und Ödeme vergrößert sich der Abstand zwischen Alveolen und Kapillaren [47]. Dies kann zu einer Diffusionsstörung beitragen, wobei Kohlendioxid aufgrund seines höheren Diffusionskoeffizienten weiterhin diffundiert, jedoch gleichzeitig die Sauerstoffdiffusion herabgesetzt ist $[49,50]$. Darüber hinaus kommt es infolge eines Lungenödems und der darauf folgenden Verkleinerung des alveolären Luftraums aufgrund der Persistenz der arteriellen Perfusion im nicht belüfteten Lungengewebe zu einem intrapulmonalen Shunt [46]. Die Hypoxämie, welche von den peripheren Chemorezeptoren in den Glomera aortica erkannt wird, verstärkt den Atemantrieb und löst eine Hypokapnie aus [49]. Bei Patienten mit einer leichten bis mittelgradigen COVID-19-Erkrankung kommt es als Reaktion auf die Hypoxämie zu einem entsprechenden Anstieg des Atemantriebs, wodurch ein normaler bis niedriger Kohlendioxidspiegel aufrechterhalten wird [3, 7, 51].

\section{Thrombosen und Mikrogerinnsel}

Thrombosen und Mikrogerinnsel sind für eine Zunahme der Beatmungs-/Perfusionsfehlanpassung und des physiologischen Totraums (VD), d.h. der normalerweise belüfteten, aber schlecht perfundierten Zonen, verantwortlich. Die Perfusionsumverteilung in das nicht betroffene Gefäßbett verringert das Verhältnis der die Alveolen erreichenden Luft pro Minute zum Herzzeitvolumen (VA/Q) und führt zur Hypoxämie. Ist die Auswirkung auf das Gefäßbett von Bedeutung und zudem das Verhältnis von Totraum zum Atemzugvolumen (VD/VT) hoch, kann dies per se die Elimination von $\mathrm{CO}_{2}$ einschränken und eine Hyperkapnie verursachen.

Es gibt Hinweise darauf, dass eine SARS-CoV-2-Infektion eine Aktivierung der Gerinnungskaskade und eine anschließende Endothelschädigung bewirkt, was zu einem prothrombotischen $\mathrm{Zu}$ stand führt $[47,52,53]$. Dies wurde häufig bei COVID-19-Patienten beobachtet $[47,54,55]$, wobei erkannt wurde, dass die Gerinnungsaktivierung und eine endotheliale Dysfunktion Prädiktoren für ein schlechteres Ergebnis und Todesfälle sind [54, 56, 57].

Ähnliche prothrombotische Reaktionen wurden bei anderen Coronaviren wie SARS-CoV-1 und MERS-CoV beobachtet [57, 58]. Diese Reaktionen stellen möglicherweise einen Versuch dar, eine diffuse alveoläre Hämorrhagie zu verhindern; durch ihre ausgedehnte Ausbildung und die Ablagerung von Fibrin verschlimmern sie jedoch die respiratorische Insuffizienz [57, 58]. Mehrere Thromben in den Lungengefäßen können durch eine Vergrößerung des funktionellen Totraums in den Alveolen die Perfusion beeinträchtigen [50].

\section{Neuroinvasion}

Wie auch andere Coronaviren besitzt SARS-CoV-2 ein neuroinvasives Potenzial und wurde in Post-Mortem-Studien im Hirngewebe nachgewiesen [59-63]. Ein möglicher Weg, über den SARSCoV-2 in das Gehirn eindringen kann, führt über den Eintritt über das Nasenepithel und anschließend über die periphere Nervenbahn über Hirnnerven $[15,64,65]$.

Ein zweiter und wahrscheinlich gleichzeitig stattfindender Mechanismus, durch den das Virus ins Gehirn gelangt, besteht im zuerst erfolgenden Eintritt in den Blutkreislauf oder das Lymphsystem über den zellulären Rezeptor Angiotensin-konvertierendes Enzym 2, der sich in den Blutgefäßen in der Schleimhaut in den Atemwegen befindet $[12,66]$, und im anschließenden Vordringen in das zentrale Nervensystem durch die Blut-HirnSchranke [66].

Ein Eintritt über den ersten Hirnnerv (olfaktorisch) wird durch die Evidenz einer ähnlichen neuralen Invasion bei anderen Coronaviren und durch Berichte über olfaktorische Symptome im frühen Krankheitsverlauf von mit SARS-CoV-2 infizierten Patienten gestützt [64]. Viele COVID-19-Patienten haben von Anosmie und Dysgeusie berichtet [67], was auch aus einer Invasion der siebten (Gesicht), neunten (Nervus glossopharyngeus) und zehnten (Nervus vagus) Hirnnerven resultieren könnte. Andererseits transportieren diese drei Nerven auch Informationen zum Nucleus des Tractus solitarius in der Medulla des Hirnstamms. Der 
Nucleus des Tractus solitarius befindet sich proximal zu den Atemzentren im Hirnstamm. Eine Beeinträchtigung dieser Nervenbahnen durch eine SARS-CoV-2-Infektion kann die Funktion der Atemzentren in der Medulla verändern und zu einer neurogenen Atemnot führen [12, 64].

Im Rahmen der neurologischen Komplikationen von COVID-19 treten Delirium und Bewusstseinsstörungen häufig auf [68-71]. Schätzungen zufolge entwickelt bis zu einem Drittel der COVID19-Patienten ein Delirium, wobei unter den Intensivpatienten ein erhöhtes Risiko und eine erhöhte Sterblichkeit bestehen, insbesondere bei älteren Erwachsenen [69-74]. Die Ätiologie des Deliriums im Zusammenhang mit COVID-19 ist aufgrund des Schweregrads der Erkrankung, der Stoffwechselveränderungen (Hypoxämie, Dehydratation und Fieber), der Einnahme von Sedativa, der sozialen Isolation und der Krankenhausumgebung, in der das Personal persönliche Schutzausrüstung trägt, multifaktoriell. Eine Neuroinvasion sollte als relevante Mitursache in Betracht gezogen werden [70, 75]. Darüber hinaus wurde bei COVID19-Patienten eine ungewöhnliche akute Enzephalopathie beschrieben [76-78]. Das klinische Bild dieser Enzephalopathie ist heterogen, reicht vom Subdelirium bis hin zum Koma, und kann sogar dem Einsetzen der Atemwegssymptome vorausgehen [77]. Der pathophysiologische Mechanismus dieser Enzephalopathie ist noch ungeklärt: Obwohl im Zusammenhang mit COVID-19 einige Fälle einer echten Enzephalitis beschrieben wurden [79], ist eine Viruspräsenz bei dieser Enzephalopathie nicht durch RTPCR nachgewiesen worden. Eine normale Anzahl an Leukozyten im Liquor deutet derweil auf einen Mechanismus hin, der von der direkten Viruspräsenz unabhängig ist [76]. Biologische Merkmale, die auf eine Störung der Blut-Hirn-Schranke hinweisen, sowie neurologische Symptome, die für eine intrazerebrale Endotheliitis sprechen, deuten möglicherweise auf einen Entzündungsmechanismus bei dieser akuten Enzephalopathie hin [78]. Dies bleibt jedoch in diesem Stadium eine Hypothese.

\section{Hypothesen zur neurologischen Veränderung der Wahr- nehmung von Atemnot bei COVID-19-Patienten}

Die Wahrnehmung von Atemnot ist bei allen Krankheitszuständen, einschließlich COVID-19, multifaktoriell. Die genauen sensorischen und neuronalen Mechanismen, die an der Wahrnehmung von Atemnot beteiligt sind, und die individuelle Reaktion auf diese Atemnot sind jedoch noch unklar $[1,16,17]$. Wie oben bereits ausgeführt, scheint die Wahrnehmung von Atemnot in jedem Krankheitszustand eine starke kognitive Komponente zu haben, was bei der Wahrnehmung von Atemnot bei COVID-19 und insbesondere bei Patienten mit akuter Enzephalopathie wahrscheinlich eine bedeutende Rolle spielt.

Das Ausmaß des Einflusses der kognitiven Komponente auf die individuelle Wahrnehmung von Atemnot unterscheidet sich wahrscheinlich bei verschiedenen Patientenuntergruppen. COVID-19-Patienten weisen eine Vielzahl von respiratorischen Phänotypen auf, einschließlich einer verminderten Sauerstoffsättigung, Tachypnoe und einer Vielzahl von intrapulmonalen $\mathrm{Pa}$ - thologien, die nicht eng mit dem Bestehen oder der Abwesenheit von Atemnot zu korrelieren scheinen [80-82]. Tatsächlich ist es möglich, dass Untergruppen von Patienten mit schwerer chronisch-obstruktiver Lungenerkrankung, wie in der klinischen Praxis beobachtet, trotz einer sehr starken Desaturierung bei Belastung oder sogar einer Hypoxämie im Ruhezustand nur eine sehr geringe Dyspnoe aufweisen. Dies wird auch bei Patienten mit anatomischen Rechts-Links-Shunts (d.h. beim EisenmengerSyndrom) festgestellt. Darüber hinaus ist das klinische Spektrum der neurologischen Symptome von COVID-19-Patienten breit gefächert und reicht von der Abwesenheit jeglicher kognitiver Symptome bis hin zum Koma.

Wir stellen die These auf, dass die Wahrnehmung von Atemnot bei COVID-19-Patienten möglicherweise aufgrund einer Schädigung der neuralen Verarbeitung der Wahrnehmung von Atemnot im Gehirn verändert ist, was an sich der kortikalen Modulation der Schmerzwahrnehmung ähnlich sein kann. Da die sensorischen und affektiven Aspekte von Atemnot durch die sensorischen und limbischen Kortexe bzw. die Inselrinde vermittelt werden, folgt daraus, dass eine Überlagerung dieser beiden Bereiche an der neuronalen Verarbeitung von Atemnot beteiligt ist. Wir stellen die These auf, dass die kortikale Verarbeitung und Modulation der Wahrnehmung von Atemnot durch SARS-CoV-2 mittels drei verschiedener Mechanismen verändert werden könnte:

1. SARS-CoV-2 könnte afferente sensorische Signalwege unterbrechen, wodurch die kortikalen Strukturen, die für die Verarbeitung der sensorischen Aspekte von Atemnot verantwortlich sind, keine afferenten Signale mehr vom Hirnstamm empfangen können. Es besteht auch die Möglichkeit, dass eine direkte Schädigung von Mechano- oder Irritant-Rezeptoren im Atmungstrakt und/oder in der Brustwand durch das Virus verhindert, dass afferente Signale den Hirnstamm erreichen und dann an höhere Hirnstrukturen weitergeleitet werden.

2. SARS-CoV-2 könnte die Fähigkeit kortikaler Strukturen beeinträchtigen, afferente sensorische Atemnotsignale vom Hirnstamm zu erkennen oder zu verarbeiten. Dies könnte sich entweder direkt durch die Wirkung von SARS-CoV-2 auf die Nervenfasern oder indirekt durch eine akute entzündliche Enzephalopathie oder zerebrovaskuläre Komplikationen wie einen ischämischen oder hämorrhagischen Schlaganfall [76, 83] ereignen.

3. Die an der Wahrnehmung von Atemnot beteiligten kortikalen Strukturen könnten, ähnlich wie bei Schmerzen, eine potenzierende Wirkung auf die Wahrnehmung von Atemnot haben, die durch SARS-CoV-2 unterbrochen werden könnte. Es hat sich gezeigt, dass eine experimentelle Inhalation von 35-prozentigem $\mathrm{CO}_{2}$ bei Patienten mit einer beidseitigen Amygdala-Schädigung von Dyspnoe ausgelöste Panikattacken verursacht, wobei die von den Patienten selbst berichteten Panik- und Angstwerte signifikant höher waren als in der neurologisch intakten Vergleichsgruppe. Dies könnte darauf hindeuten, dass $\mathrm{CO}_{2}$ Gehirnstrukturen außerhalb des limbischen Systems direkt aktiviert, welche dann von der Amygdala herunterreguliert werden.
10

Kompass Pneumol 2022;10:6-14 DOI: $10.1159 / 000521460$ 
Abb. 1. Zeitleiste zur Ausbildung von Atemnot bei Patienten mit COVID-19-Pneumonie. Im frühen Krankheitsverlauf bestehen neurologische Symptome wie Anosmie und Hypogeusie; diese spiegeln eine mögliche Neuroinvasion von SARSCoV-2 wider. Bei Patienten, die einen schweren COVID-19-Verlauf entwickeln, zählen Entzündungen in der Lunge, Diffusionsanomalien, Thrombosen und eine Diskrepanz im Ventilations-Perfusions-Verhältnis, die im Allgemeinen nach den ersten 7 Tagen der Infektion auftreten, zu den physiologischen Veränderungen. Atemnot tritt in diesem Stadium verstärkt auf und kann in der Post-COVID-Phase bestehen bleiben.

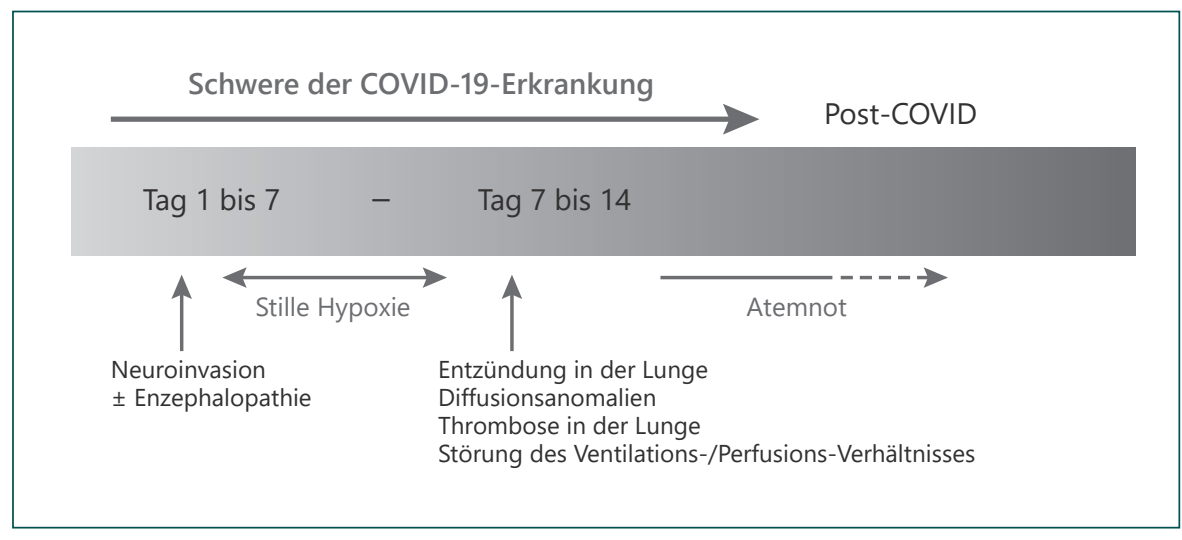

Die absolute oder relative Bedeutung eines der von diesen drei als These aufgestellten Mechanismen ist noch unklar, und jede Kombination dieser drei Mechanismen könnte einen Teil zur gegenständlichen Problematik beitragen. Darüber hinaus kann, wie bereits in den oben genannten Hypothesen diskutiert, auch das unterschiedliche Ausmaß von Lungen- und Hirnschädigungen zu einer veränderten Wahrnehmung von Atemnot beitragen [84]. Ebenso ist jedoch klar, dass es bei vielen COVID-19-Patienten zur Atemnot kommt und dass die Prävalenz der Atemnot bei Patienten mit schwererer Lungenerkrankung und mit schlechterer Prognose höher zu sein scheint $[80,81,85]$. Hierzu stellen wir die Hypothese auf, dass die direkten neuroinvasiven Auswirkungen von SARS-CoV-2 die Wahrnehmung von Atemnot durch einen oder mehrere der drei oben als These aufgestellten Mechanismen abschwächen oder herabsetzen könnten. Im Gegensatz dazu schädigen indirekte Auswirkungen von SARS-CoV-2 auf das Herz-Kreislauf-System, die interstitielle Entzündungen und Thrombosen verursachen, die Atmungsfähigkeit der Lunge, was letztendlich zum Versagen des Gasaustauschs und zum Organversagen führt.

Wir stellen die These auf, dass Zeit hierbei eine Rolle spielt, wobei neuronale Schäden und der Verlust der Wahrnehmung von Atemnot im frühen Stadium der Erkrankung vorherrschen. Da die neurologischen Komplikationen wie die akute Enzephalopathie den respiratorischen Symptomen sogar vorausgehen können, können wir die Hypothese aufstellen, dass das Phänomen der «glücklichen» oder «stillen Hypoxie» ein präklinisches Symptom des Virus darstellen könnte. Bei Patienten mit nur leichter Erkrankung herrscht dieser Phänotyp während des gesamten Krankheitsverlaufs vor. Wenn es zur Atemnot kommt, wie dies im Fall von schweren Krankheitsverläufen auffällt, tritt diese erst später auf, wenn sich bereits eine kardiopulmonale Schädigung entwickelt hat [14]. Diese Zeitachse ist in Abbildung 1 dargestellt. Schließlich stellen wir die These auf, dass der Einfluss von SARSCoV-2 auf die zentrale neuronale Verarbeitung von Atemnot am entgegengesetzten Ende eines Gleichgewichts mit den Auswirkungen von kardiorespiratorischen Entzündungen und Thrombosen liegt. Bei Patienten mit leichter Erkrankung oder im frühen Krankheitsstadium herrscht aufgrund der Störung der Gehirn-

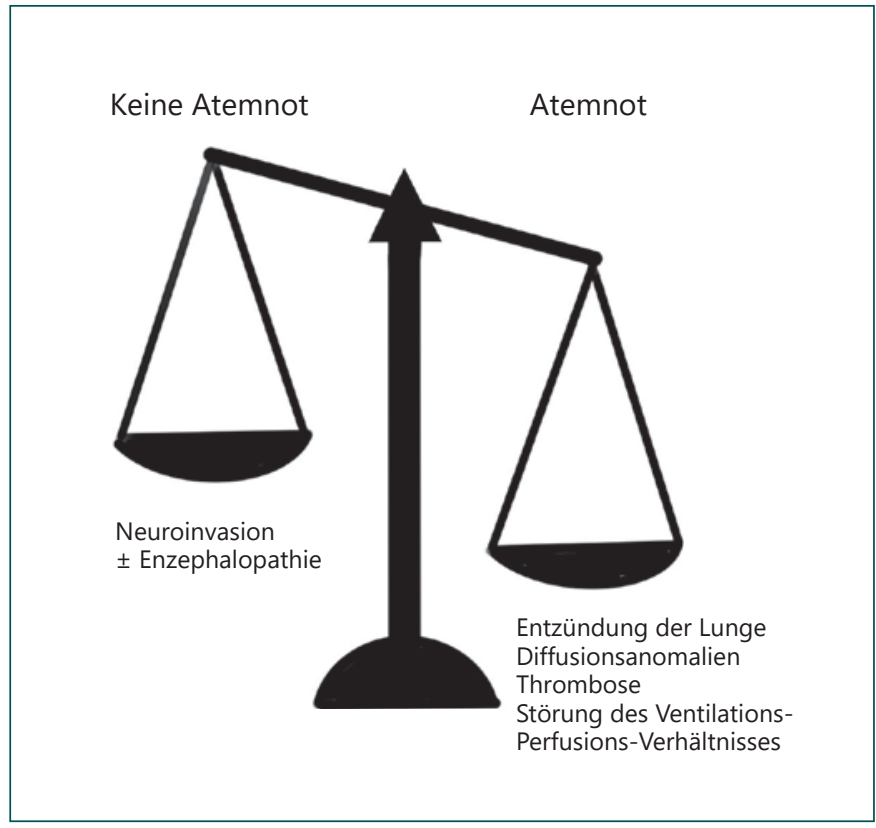

Abb. 2. Atemnot bei COVID-19: ein Gleichgewicht aus verschiedenen Faktoren, die die Symptomwahrnehmung beeinflussen. Neuroinvasion und Enzephalopathie scheinen die Atemnot in der frühen Krankheitsphase zu verringern. In schwereren Fällen erhalten Entzündungen, Diffusionsveränderung, Thrombosen und Störungen des Ventilations-Perfusions-Verhältnisses im weiteren Krankheitsverlauf ein stärkeres Gewicht als die Veränderung bei der Wahrnehmung von Atemnot, die von der Neuroinvasion von SARS-CoV-2 verursacht wird, und die Patienten werden zunehmend kurzatmig.

funktion durch SARS-CoV-2 die Verringerung der Wahrnehmung von Atemnot vor, was zum scheinbaren Widerspruch zwischen dem physiologischen Zustand und der Atemnot führt. Bei schwer erkrankten Patienten herrscht wegen der Schädigung der Atmungsfähigkeit die Atemnot vor, da der Körper die physiologischen Signale, die sich aus dem drohenden Organversagen ergeben, nicht mehr ignorieren kann. Es ist wahrscheinlich, dass die Wahrnehmung von Atemnot mit abnehmender Lungenfunktion zunimmt. Die am stärksten Betroffenen können ihre Symptome nicht mehr angeben, da sie bereits unter Sedierung beatmet werden (siehe Abb. 2). 


\section{Perspektiven und Forschungsmöglichkeiten}

\section{Diskussion}

An der Pathophysiologie von Atemnot ist ein Zusammenspiel aus mehreren komplexen Mechanismen in der Interaktion zwischen Gehirn und Lunge beteiligt. Dazu zählen mechanische, neurologische und kognitive Prozesse. Auch individuelle, nicht mit COVID-19 in Zusammenhang stehende Faktoren des jeweiligen Patienten wie Lebensalter und Begleiterkrankungen wie Diabetes spielen eine Rolle, ebenso wie soziale und Umweltfaktoren. Im Falle von COVID-19 wurde die Abwesenheit der Wahrnehmung von Atemnot bei hypoxischen Patienten weithin beobachtet, was die Hintergründe dieses Symptoms noch unergründlicher macht.

Wie bereits erwähnt, ist die Bandbreite der Atemnot, über die in Studien berichtet wurde, groß. Ein Teil dieser Verschiedenartigkeit lässt sich durch die unterschiedlichen Methoden erklären, die zur Evaluierung der Atemnot herangezogen werden; diese wurden nämlich in der Mehrzahl der Studien, die sich mit der Symptomprävalenz bei COVID-19 befassten, nicht angegeben. Daher könnten unterschiedliche Methoden, etwa die modifizierte BorgSkala oder die numerische Rating-Skala, verwendet worden sein [86]. Es ist jedoch auch möglich, dass Atemnot gemäß der Einschätzung der behandelnden Ärzte oder Forscher auf Basis von objektiven klinischen Anzeichen wie Tachypnoe, Mimik oder dem Einsatz der Atemhilfsmuskulatur in den Krankenakten dokumentiert wurde. Dyspnoe ist subjektiv, und durch körperliche oder klinische Anzeichen können die Beschwerden der Patienten unter- oder in diesem Fall überschätzt werden [87]. Darüber hinaus war die Schwere der Atemnot in Metaanalysen nicht dokumentiert, sie war entweder vorhanden oder sie fehlte, sodass die klinische Auswirkung der Atemnot nicht bewertet werden konnte. Schließlich ist Atemnot ein häufiges Symptom in der Allgemeinbevölkerung, und Patienten können eine COVID-19-Erkrankung entwickeln und zu diesem Zeitpunkt schon an Atemnot leiden. Dies könnte zu einer Überschätzung der Anzahl der Patienten führen, die im Zusammenhang mit COVID-19 an Atemnot leiden. Stattdessen könnte daher die Anwendung von Abweichungen bei der Atemnot einen genaueren Maßstab für die von COVID-19 verursachte Atemnot darstellen und sollte insofern in weiteren Studien erwogen werden.

Wir haben auf die aktuell verfügbare Literatur zurückgegriffen, um die Mechanismen der Wahrnehmung von Atemnot sowie die Umstände zu diskutieren, wie diese während einer SARS-CoV2-Infektion beeinflusst werden können. Dazu zählen Entzündungen des Lungengewebes, die Entwicklung von Mikrothromben im Lungenkreislauf sowie neurologische Komplikationen wie zerebrovaskuläre Ereignisse oder eine akute Enzephalopathie. Wir haben drei zusätzliche Haupthypothesen aufgestellt, die auf neuropsychologischen Prozessen basieren, um das Phänomen der verminderten Wahrnehmung von Atemnot zu erklären, das bei COVID-19-Patienten beobachtet wurde, sowie um Thesen über Zusammenhänge zwischen kognitiven und physiologischen Komponenten bei der Atemnot und deren Wechselwirkungen im
Laufe der Zeit aufzustellen. Um diese Hypothesen zu bestätigen, ist jedoch eine weitere Evidenz erforderlich.

Jüngste Berichte haben die Prävalenz von Dyspnoe bei Nachsorgeuntersuchungen nach einer COVID-19-Erkrankung verdeutlicht [88]. Dabei ist die Feststellung interessant, dass noch $2 \mathrm{Mo}$ nate nach Auftreten der ersten COVID-19-Symptome Atemnot und Erschöpfung angegeben werden, obwohl die meisten Symptome im Laufe der Zeit zu verschwinden scheinen [89].

Obschon gezeigt wurde, dass der Schweregrad der Post-COVID19-Symptomatik mit der Schwere der akuten COVID-19-Phase in Zusammenhang steht, ist noch nicht klar, bei welchen Patienten eine persistierende Dyspnoe auftreten wird [88]. Viele Patienten litten an Kurzatmigkeit und anderen damit zusammenhängenden Symptomen, die nach der anfänglichen Phase der akuten Infektion beginnen oder darüber hinaus bestehen bleiben, etwa chronischer Husten, Engegefühl in der Brust, kognitive Dysfunktion und Erschöpfung, die allgemein als «Long-COVID» bezeichnet werden. Es ist noch nicht bekannt, ob sich bei Patienten, bei denen in der akuten COVID-19-Phase keine Dyspnoe auftrat, nach dem Ende der Erkrankung schließlich eine Dyspnoe entwickeln wird. Dies könnte darauf hindeuten, dass SARS-CoV-2 möglicherweise einen Einfluss auf die Wahrnehmung von Atemnot hat. Eine alternative Hypothese zur Erklärung dieser aufkommenden Atemnot bei Patienten, die während der akuten Phase nicht an einer Dyspnoe litten, könnte mit den komplexen und noch nicht geklärten pathophysiologischen Mechanismen von Long-COVID zusammenhängen, etwa den immunologischen und Entzündungsreaktionen auf die akute Infektion.

Erschöpfung steht in engem Zusammenhang mit Atemnot, und die Mechanismen der von COVID ausgelösten Erschöpfung lassen sich möglicherweise extrapolieren, um so unser Verständnis von Atemnot im Zusammenhang mit COVID zu verbessern. Beispielsweise scheint das Vorhandensein von geistiger und körperlicher Erschöpfung mit oder ohne Dysexekutionssyndrom auf die Auswirkungen von COVID-19 auf die Hirnfunktion zurückzuführen zu sein. Die Fachliteratur legt nahe, dass Erschöpfung durch eine funktionelle Beeinträchtigung der inhibitorischen Hauptkreise im primär-motorischen Kortex hervorgehoben werden könnte, was durch transkranielle Magnetstimulation nachgewiesen wurde [90]. In den Erkenntnissen der transkraniellen Magnetstimulation ist eine Evidenz dafür ersichtlich, dass COVID-19 möglicherweise zu einer Beeinträchtigung der GABAergen intrakortikalen Kreise führt, und es ist möglich, dass COVID-19 ähnliche Auswirkungen auf die kortikalen Bahnen haben könnte, die im Zusammenhang mit Atemnot stehen [90-92].

Obwohl sich in Berichten gezeigt hat, dass für Patienten in der Post-COVID-19-Phase die Gefahr einer Entwicklung chronischer Schmerzen besteht [93, 94], wurde kürzlich darauf hingewiesen, dass eine akute SARS-CoV-2-Infektion die nozizeptive Schmerzwahrnehmung reduzieren kann [95]. Obwohl die Evidenz in diesem Stadium noch mangelhaft ist, hat sich gezeigt, dass SARS-CoV-2 in Tiermodellen die neuropathischen Schmerzsignale durch Bindung an Neuroliptin-1-Rezeptoren auf sensorischen Neuronen hemmt [96]. In diesem Fall scheint die Schmerz- 
modulation von peripheren Nerven und nicht von intrazerebralen Signalen auszugehen. Obwohl diese Ergebnisse noch bestätigt werden müssen, können sie nützliche Erkenntnisse darüber darstellen, wie dieses Virus nicht nur Schmerzen, sondern möglicherweise auch die Atemnot beeinflusst.

\section{Einschränkungen}

COVID-19 ist eine neue Krankheit, und unser Wissensstand über sie entwickelt sich schnell und beständig weiter. Unser Artikel unterliegt daher der Einschränkung auf das bereits bekannte Wissen aus der bereits verfügbaren veröffentlichten empirischen Literatur und der kleinen Anzahl an Post-Mortem-Studien. Darüber hinaus haben die Autoren keine Qualitätsbewertung der in diesem Artikel vorgestellten Literatur durchgeführt.

\section{Implikationen für die klinische Praxis}

In das Phänomen der verminderten Wahrnehmung von Atemnot bei COVID-19-Patienten sind Mechanismen involviert, die möglicherweise zur zukünftigen Verbesserung der Behandlung von Dyspnoe genutzt werden können. Die Vertiefung unseres Wissens über die physiologischen Bahnen, die bei verschiedenen $\mathrm{Pa}$ tienten zu einer unterschiedlichen Wahrnehmung von Atemnot führen, kann eine Differenzierung zwischen denjenigen Patienten ermöglichen, bei denen eine Ausbildung oder ein Erleben von Atemnot mehr oder weniger wahrscheinlich ist, und einen besseren Einsatz von begrenzten Ressourcen erlauben. Besondere Aufmerksamkeit sollte den Patienten gewidmet werden, die keine Atemnot verspüren, da sich ihr klinischer Zustand schnell verschlechtern könnte.

Die Reaktion auf die COVID-19-Pandemie erstreckte sich auf einen multidisziplinären Ansatz, der eine multiqualifizierte Taskforce umfasst, zu der Personal aus den Fachgebieten Innere Medizin, Geriatrie, Beatmungsmedizin, Neurologie, Primär-, Intensiv- und Palliativmedizin sowie Pathologie zählt. Durch die Förderung einer integrierten Versorgung dieser Disziplinen bot die COVID-19-Pandemie die Möglichkeit, Verbindungen zwischen diesen Fachgebieten aufzubauen.

Die hier vorgestellte Literatur hat Auswirkungen auf die gesamte multidisziplinäre Taskforce. Genau dieser integrierte Ansatz wird beim Aufbau unseres Verständnisses dieser Erkrankung sowie der komplexen Physiologie, die ihren Symptomen zugrunde liegt, benötigt. Dies wird auch Auswirkungen auf Nicht-COVID19-Patienten haben, einschließlich Patienten mit Krebserkrankungen, Lungenerkrankungen, Herzinsuffizienz und neurodegenerativen Erkrankungen, die ähnliche quälende Atemnotsymptome erleben.

\section{Implikationen für die Forschung}

Durch die Schaffung einer ungewöhnlichen Symptomantwort stellt COVID-19 unser Verständnis der Mechanismen, die der Atemnot zugrunde liegen, und ihrer möglichen Bahnen infrage und bietet uns eine Gelegenheit zur Erforschung der Behandlung eines Symptoms, das sowohl belastend als auch nur schwer behandelbar ist. Es gibt keinen Goldstandard zur Messung von Atemnot
[97]. Das validierte Instrumentarium kann in drei Kategorien eingeteilt werden. Eindimensionale Methoden zur Evaluierung von Atemnot wie die visuelle Analogskala, die numerische Rating-Skala oder die modifizierte Borg-Skala werden häufig angewandt. Diese eindimensionalen Skalen sind jedoch dadurch eingeschränkt, dass in ihnen nur die Intensität der Dyspnoe erfasst wird. Multidimensionale Methoden ermöglichen hingegen eine tiefgreifendere Bewertung, da in ihnen auch andere Bereiche wie körperliche, emotionale oder weitere Dimensionen der gesundheitlichen Lebensqualität einbezogen werden können; darunter fallen die Dyspnoe-12-Skala oder Dyspnoe-Skalen aus krankheitsspezifischen Instrumenten wie der Chronic Respiratory Questionnaire [98-100]. Andere Methoden ermöglichen eine Bewertung spezifischer Dimensionen, etwa die Medical Research Council Dyspnoea Scale, die die Aktivitätseinschränkung im Zusammenhang mit Atemnot misst [97, 101, 102]. Obwohl es sich bei Dyspnoe um ein subjektives Symptom handelt, sind Instrumente, bei denen keine Patientenbeteiligung erforderlich ist, validiert und sollten Anwendung finden [103]. Darüber hinaus sollte auch eine gründlichere Untersuchung der Korrelation zwischen den Angaben der Patienten zur Atemnot und der mittels Blutgasen sowie biologischen und physikalischen Markern gemessenen Krankheitsschwere in Betracht gezogen werden.

Die in diesem Artikel vorgestellte Evidenz ist umfangreich, wobei wichtige Überlegungen beschrieben werden, die für ein multidisziplinäres Publikum relevant sind. Daher ist es möglich, mehrere Fragen für die Forschung vorzuschlagen, die sich sowohl auf pathophysiologische als auch auf patientenbezogene Faktoren erstrecken und für eine Vielzahl von Disziplinen von Bedeutung sind. In der weiteren Forschung sollte die Verwendung validierter uni- und multidimensionaler Dyspnoe-Messinstrumente erwogen werden, um die Entwicklung der Atemnotintensität im Krankheitsverlauf und in der Post-COVID-Phase zu quantifizieren. Angesichts des neuroinvasiven Potenzials von SARS-CoV-2 könnten funktionelle MRTs verwendet werden, um ein tieferes Verständnis des Unterschieds in der bei COVID-19-Patienten beobachteten Gehirnaktivität und ihres Beitrags zur Verschiedenartigkeit der Wahrnehmung von Atemnot zu erlangen. Schließlich sollten Pathologen und Grundlagenforscher die neurotropen Mechanismen von SARS-CoV-2 sowie ihren möglichen Einfluss auf die Wahrnehmung von Atemnot weiter untersuchen.

\section{Fazit}

Atemnot ist ein häufiges und lähmendes Symptom bei Patienten im fortgeschrittenen Krankheitsstadium. Nachdem nun in mehreren Berichten aufgezeigt wurde, dass Atemnot bei COVID19-Patienten trotz schwerer physiologischer und anatomischer Veränderungen in der Lunge häufig gering ausgeprägt ist oder überhaupt nicht besteht, ist deutlich geworden, dass COVID-19 die Wahrnehmung von Atemnot beeinflusst.

Da Patienten mit leichter COVID-19-Erkrankung durch eine Steigerung ihres Atemantriebs immer noch eine physiologische Reaktion auf Hypoxämie zeigen, vermuten wir, dass an der Ver- 
änderung der Wahrnehmung von Atemnot höhere kortikale Strukturen beteiligt sind, die entweder die Interpretation afferenter Signale direkt beeinflussen oder die Top-down-Regulierung von Atemnot verändern. Darüber hinaus sind wir der Ansicht, dass eine starke Korrelation zwischen Atemnot und der fortschreitenden Schädigung der Lunge besteht.

Derzeit sind weiterhin viele Fragen unbeantwortet und in $\mathrm{Zu}$ kunft sollte sich die Forschung darauf konzentrieren, unsere Kenntnisse dieses Phänomens zu schärfen und zu verbessern. Ein multidisziplinärer Ansatz wird entscheidend sein, um Erkenntnisse über die pathophysiologischen Mechanismen zu gewinnen, die an der Veränderung der Atemnot bei COVID-19-Patienten beteiligt sind.

\section{Danksagung}

Die Autoren möchten sich bei allen Patienten und ihren Angehörigen bedanken, die beim Thema COVID-19 zum Wissenszuwachs beitragen.

\section{Erklärung zu Interessenskonflikten}

Bei den Autoren bestehen keine Interessenskonflikte, die zu melden wären.

\section{Finanzierungsquellen}

Für diese Forschung wurden keine spezifischen Zuschüsse von Fördereinrichtungen aus dem öffentlichen, kommerziellen oder gemeinnützigen Sektor erhalten.

\section{Beiträge der Verfasser}

LH, SC, IS, RE und EA haben das Manuskript geschrieben. Alle Autoren haben die Literatur studiert und das Manuskript bearbeitet und kommentiert. SC hat die Abbildungen erstellt. Alle Verfasser haben das zu veröffentlichende Manuskript in der finalen Fassung gelesen und genehmigt.

\section{Literatur}

Die Literatur ist unter www.karger.com/Article/Fulltext/521460 abrufbar. 